\title{
Coronavirus Disease 2019: Psychological Stress and Cardiovascular Diseases
}

\author{
Maki Komiyama (D) and Koji Hasegawa (D)
}

Division of Translational Research, National Hospital Organization Kyoto Medical Center, Kyoto, Japan

\begin{abstract}
Minimising deaths due to coronavirus disease 2019 (COVID-19) is a global priority. However, the harmful effects are not limited to those directly related to the infection. The COVID-19 pandemic has also had a serious impact on the mental health of the general population. An increasing number of people are exhibiting signs of depression and an increase in suicides has also been noted around the world. Mental health issues may be linked to starting or increasing the use of addictive substances, such as tobacco, alcohol and drugs, along with increased overweight and obesity resulting from changes in eating habits. These issues can impact cardiovascular diseases because of worsened risk factor control. This review discusses the impact of the COVID-19 pandemic on mental health and cardiovascular risk factors. It will also summarise the measures that can be taken to maintain good mental health and their importance in mitigating cardiovascular disease.
\end{abstract}

\section{Keywords}

COVID-19, psychological stress, depression, mental health, smoking, obesity, cardiovascular disease

Disclosure: $\mathrm{KH}$ is on the European Cardiology Review editorial board; this did not influence peer review. MK has no conflicts of interest to declare.

Received: 23 March 2021 Accepted: 4 May 2021 Citation: European Cardiology Review 2021;16:e33. Dol: https://doi.org/10.15420/ecr.2021.10

Correspondence: Maki Komiyama, Clinical Research Institute, National Hospital Organization Kyoto Medical Center, 1-1 Mukaihata-cho, Fukakusa, Fushimi-ku,

Kyoto 612-8555, Japan. E: nikonikomakirin@yahoo.co.jp

Open Access: This work is open access under the CC-BY-NC 4.0 License which allows users to copy, redistribute and make derivative works for non-commercial purposes, provided the original work is cited correctly.

The coronavirus disease 2019 (COVID-19) pandemic is ongoing and has had a severe impact around the world. The pandemic has been classified as a public health problem that needs to be dealt with urgently, and minimising deaths due to the virus is an international priority. However, the harmful effects reach beyond those caused by the infection itself. COVID-19 has also had a serious impact on the mental health of the general population. An increasing number of people are exhibiting signs of depression globally, and an increase in suicides has been noted. Mental health issues may be associated with starting or increasing the use of addictive substances, such as tobacco, alcohol or drugs, along with changes in eating habits. These factors may in turn exacerbate cardiovascular risk.

\section{COVID-19-related Depression}

Various countries have been forced to implement strict systems of hygiene and social distancing because of the COVID-19 pandemic leading to their populations dealing with stressful events. The pandemic has caused a mental health crisis, as evidenced by the appearance of the new term 'COVID-19-related depression'? The WHO has highlighted prolonged physical and social isolation due to lockdowns, fear of infection and losing cherished family and friends, an economic crisis, an increase in domestic violence and abuse primarily of women and children along with anxiety about young persons' futures following disruption of their education as factors contributing to this crisis. Furthermore extensive misinformation about the virus and preventive measures and repeated images in the media of seriously ill people can intensify fear.'

Typically, mental health issues exist on a continuum from a minor issue lasting a certain period of time to more serious issues. ${ }^{2}$ The COVID-19 pandemic has affected everyone's mental health and the state of people with prior mental health issues has worsened, leading to dysfunction. ${ }^{2}$ Citing the Lancet Commission on Global Mental Health and Sustainable Development, the UN warned: "Many people who previously coped well, are now less able to cope because of the multiple stressors generated by the pandemic."3

In fact, symptoms of depression or anxiety are reported to be at higher levels than normal in various countries. According to the UN, surveys after the start of the pandemic indicated that $35 \%$ of people in China, $45 \%$ of people in the US, and $60 \%$ of people in Iran had their mental health troubled by the COVID-19 pandemic. ${ }^{3}$ The US Centers for Disease Control and Prevention conducted an online survey of 5,412 American adults in June 2020 to assess their mental health, substance use and suicidal ideation during the pandemic. ${ }^{4}$ Of those respondents, $40.9 \%$ indicated that they had some form of a psychiatric disorder, including an anxiety disorder or symptoms of a depressive disorder, and $10.7 \%$ indicated that they had seriously considered suicide in the past 30 days. ${ }^{4}$ This was a substantial increase compared to the same period in 2019.

A large-scale study was conducted in the Amhara region of Ethiopia in April 2020, finding that the estimated prevalence of symptoms matching a depressive disorder was $33 \% .{ }^{5}$ This was a threefold increase compared to the level before the outbreak. A study in adults in the US reported that symptoms of depression posed a bigger burden on subjects with lower income or few savings and on subjects who were often exposed to stressors, such as loss of a job. ${ }^{6}$ The negative mental health impacts associated with the COVID-19 pandemic have also been reported to be more pronounced for women than men among people living in rural areas 
Figure 1: The Relationship Between COVID-19-related Stress and Smoking

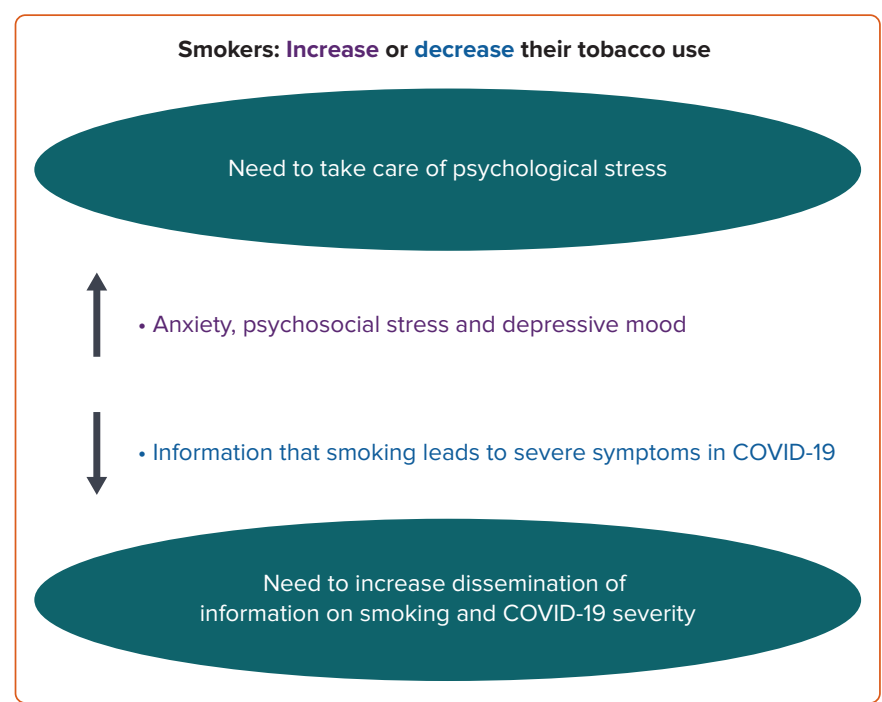

COVID-19 = coronavirus disease 2019 .

because of increased care responsibilities and loss of income. In Japan, suicides increased for 4 months consecutively after July 2020, and overall suicides in October 2020 increased $39.9 \%$ compared to the same period the previous year. Of note, suicides in women increased by $82.6 \%$ in October 2020 compared to October 2019. ${ }^{8}$

\section{Stress and Dependence as a Result of COVID-19}

Prolonged confinement following a large-scale natural disaster is known to greatly affect people mentally, as exemplified by an increased incidence of psychiatric disorders following the imposed lockdown measures. ${ }^{9}$ Being subjected to stress in conditions with no known time limit, such as when a state of emergency is declared, can lead to an inability to control one's decision-making. ${ }^{10}$

To cope with stress, people tend to rely on negative coping strategies, such as the use of addictive drugs, alcohol or tobacco, or spending excessive time engaging in potentially addictive behaviours, such as online gaming. Stress is a well-known risk factor for the development and worsening of dependence and vulnerability to its recurrence.

The underlying pathophysiology is the effects of those changes (i.e. substance use or addictive behaviour) on corticostriatal-limbic systems of motivation, learning and adaptation, including the mesolimbic dopamine, glutamate and $\gamma$-aminobutyric acid pathways. ${ }^{11}$ There are global concerns about an increase in the use of addictive substances, such as alcohol, to cope with stress associated with self-isolation amidst the COVID-19 pandemic. ${ }^{12}$ According to Canadian statistics, alcohol consumption among people aged $15-49$ years increased $20 \%$ during the pandemic. ${ }^{13}$ A study in the US reported that $13.3 \%$ of adults aged 18 or over had started or increased the use of drugs. ${ }^{4}$ Worsening health behaviour change has also been reported to be associated with being younger, female and having a higher BMI. ${ }^{14}$

\section{The Relationship Between Stress and Smoking as a Result of COVID-19}

COVID-19 has had a similar impact on smoking. Stress and worsening mental health are known to be predisposing factors for increased nicotine dependence among smokers, increased smoking (both in quantity and frequency) and resumption of smoking. ${ }^{15}$ When a state of emergency is declared, time spent at home increases as a result of self-isolation and smoking habits change as a result. ${ }^{15-17}$ Various studies to examine this are being conducted in different countries. Numerous studies have reported an increase in the number of cigarettes smoked, but conversely, other studies have also reported no change in the total number of cigarettes smoked since some people smoke more while others smoke less..$^{15-17} \mathrm{~A}$ study in the UK found that $25.2 \%$ of current smokers $(n=329)$ smoked more than usual, $50.9 \%$ smoked the same amount and $20.2 \%$ smoked less. ${ }^{17}$

At present, no studies have examined which factors predict smoking behaviour - either an increase or decrease in the number of cigarettes - during the COVID-19 pandemic. However, it has been reported that high depressive tendency is closely related to the failure to quit smoking. ${ }^{18}$ Consequently, smokers with existing depressive tendencies or with worsened signs of depression due to the pandemic may be causing an increase in the number of smokers. ${ }^{15,17}$ In fact, that study noted a significant worsening of mental health $(p<0.001)$, anxiety $(p<0.001)$, stress $(p<0.001)$ and a depressed mood ( $p=0.012$ ) in smokers who smoked more. ${ }^{17}$ It also revealed that worsening mental health and psychosocial wellbeing due to the pandemic was associated with increased smoking. Figure 1 shows suggested relationships between COVID-19-related stress and smoking.

For tobacco companies, this represents a golden business opportunity, and their promotional campaigns in the media are not stopping. ${ }^{19,20}$ Caution is warranted, since tobacco use may increase and the number of people who resume smoking may also increase. Caution is also warranted since having fewer social ties is associated with starting to smoke. ${ }^{21}$ That said, the fact that some people are smoking less warrants attention. The threat of contracting COVID-19 and developing severe symptoms has motivated people to improve their health. ${ }^{22}$ Moreover, greater awareness of health risks, reduced availability and socialising could reduce consumption. ${ }^{23}$ Smoking is a key factor for developing more severe symptoms of COVID-19 and is a serious threat to smokers. ${ }^{24}$ Given the demonstrated harm of smoking and exposure to second-hand smoke, the WHO recommends that people quit smoking. ${ }^{25}$ Smoking less is ineffective, but quitting can lead to restoration of immune functions and reduced susceptibility to infection in as little as a month. Providing smokers with this information could further encourage them to quit smoking.

\section{Mental Health Improves as a}

\section{Result of Quitting Smoking}

The reduction in stress that smokers view as a benefit of smoking is ultimately nothing more than the alleviation of the symptoms of nicotine withdrawal. In sharp contrast, quitting smoking is known to eliminate symptoms of withdrawal, decrease stress and alleviate psychiatric disorders, such as anxiety neurosis and depression, over the long term. ${ }^{26}$ A Japanese study reported that depressive symptoms were significantly alleviated in a brief period of just 12 weeks after the start of smoking cessation treatment. ${ }^{27}$ The extent to which depression is alleviated by quitting smoking is equivalent to or greater than the extent to which mood or anxiety disorder is alleviated by drug therapy (antidepressants). ${ }^{26}$ Now that the COVID-19 pandemic has brought serious mental health issues to light, quitting smoking will be increasingly important.

\section{Methods of Quitting Smoking During the COVID-19 Pandemic}

Numerous reviews have assessed interventions to help people quit smoking. A combination of smoking cessation aids and behavioural 
support should be used to maximise the percentage of individuals who successfully quit smoking. ${ }^{28}$

A study in a smoking cessation clinic in Japan reported that a high percentage of individuals successfully quit smoking at the end of five visits; $82 \%$ were using nicotine patches and $83 \%$ were taking varenicline. ${ }^{29}$ Therefore, an important duty for medical personnel is to encourage smokers who are hesitant about quitting smoking to visit a smoking cessation clinic. It is also necessary to be aware that the increased psychological stress may diminish smokers' recognition of the extensive established benefits of smoking cessation. Although there are debates regarding the infection rates in smokers and non-smokers, smoking has been established as a risk factor for disease severity. ${ }^{24,25}$

Furthermore, it is well known that quitting smoking restores immunity in a few weeks. Therefore, it is important for healthcare professionals to continue providing smokers information that smoking is a factor for the development of more severe symptoms of COVID-19. When seeing patients, medical personnel should also ask about and record smoking status. Mental healthcare may be even more important when providing smoking cessation support amidst the pandemic. Additionally, behavioural support provided via printed materials from reliable services, online programmes and mobile phone text messaging apps is considered to be effective. In many countries, smokers can also receive advice on quitting smoking via a quit line. In an age of reduced travel outside the home, services that can be provided remotely are increasingly important. ${ }^{30}$ Public education about quitting smoking should be highly encouraged as part of public health efforts to reduce the spread of COVID-19.

\section{Quarantine and Weight Gain}

Lifestyles have significantly changed in light of the COVID-19 pandemic. People have gained weight during the pandemic due to lack of exercise and increased calorie intake as a result of self-quarantine and stress. Additionally, an increase in sedentary behaviour has a negative influence on mental health. ${ }^{31}$ Stress is known to cause weight gain. Appetite is greatly affected by internal factors, such as an empty stomach, as well as external factors, such as dietary preferences and environmental stimuli. When the body is subjected to stress, cortisol is secreted by the adrenal glands, which stimulates appetite. Not only can this lead to overeating, but also excess energy as a result of the action of cortisol is stored as fat.

A cross-sectional online survey of 1,097 people examined the effects of the COVID-19 pandemic on diet and weight changes and found that more than $43.0 \%$ of the respondents ate more and that close to $52 \%$ snacked more. ${ }^{32}$ Those trends were even more evident in overweight and obese respondents. Moreover, $30 \%$ of the respondents reported weight gain $(3.0 \pm 1.6 \mathrm{~kg})$. Weight gain was associated with reduced consumption of fresh foods and legumes and increased consumption of processed foods with high fat, sugar or salt content due to restrictions and lockdowns. Conversely, over $18 \%$ of the respondents reported weight loss $(-2.9 \pm$ $1.5 \mathrm{~kg}$ ), and underweight subjects reported further weight loss, prompting concerns about conditions, such as sarcopenia. Overeating and smoking are closely linked to psychological stress. Salivary cortisol levels - a marker of psychological stress - have been reported to be high in obese patients who have difficulty losing weight. ${ }^{33}$

During the COVID-19 pandemic, people who were originally overweight and obese have experienced greater weight gain. ${ }^{32}$ Therefore, it is considered that people who have greater psychological stress and tend to be obese are gaining weight due to further increased stress.
Figure 2: The Effects of Psychological

Stress on Cardiovascular Diseases During the COVID-19 Pandemic

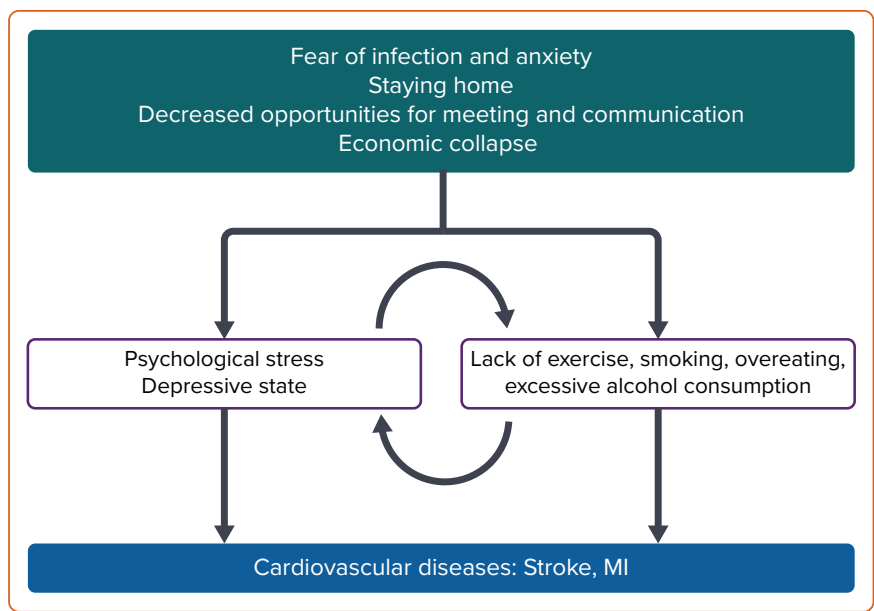

COVID-19 = coronavirus disease 2019 .

Incidentally, $14.6 \%$ of the respondents in this study reported increased alcohol consumption, and in particular people with alcoholism reported increased alcohol consumption. ${ }^{32}$ Overweight and obesity are largely preventable by making the choice of healthier foods, limiting energy intake from fats and sugars and increasing consumption of fruit and vegetables, along with legumes, whole grains and nuts. ${ }^{34}$ In addition to these dietary instructions, engagement in regular physical activity will increase effectiveness, as it not only improves overweight but also reduces psychological stress.

Worldwide, 3 million deaths every year result from harmful use of alcohol, accounting for $5.3 \%$ of all deaths. ${ }^{35}$ The WHO advocates strategies for reducing harmful alcohol use. ${ }^{36}$ There are also epidemiological data that low-volume drinkers have less cardiovascular disease than non-drinkers. ${ }^{37}$ At present, international measures regarding alcohol control measures are not as thorough as tobacco control measures. While excessive alcohol consumption clearly contributes to an increase in diseases including cancer, liver disease and cardiovascular disease, there remain many people with potential alcoholism. An increase in drinking volume during the COVID-19 pandemic may be the result of psychological stress along with overeating and smoking. In patients with cardiovascular disease, it is important to check drinking levels and carefully observe the possibility of alcohol addiction.

Lockdowns imposed to contain an infectious pathogen have multiple effects on lifestyles throughout the population. Restrictions and lockdowns lead to decreased physical activity, unhealthy diets due to restricted access to healthy and fresh food, restricted access to health promotion services, worsening mental health and misuse of tobacco and alcohol leading to secondary health hazards (Figure 2). There is mounting evidence that obesity is a risk factor for cardiovascular diseases, and for developing severe pneumonia due to COVID-19. ${ }^{38}$ Obesity inhibits breathing, weakens immunity and enhances the inflammatory response. Although there are no guidelines advocating weight loss as a way to deal with COVID-19, prevention of obesity is recommended.

\section{Effects of the Pandemic on Cardiovascular Diseases}

The COVID-19 pandemic may trigger the onset of or exacerbate cardiovascular diseases and caution is warranted. Moreover, the COVID-19 
pandemic is causing serious mental health issues, and the association between depression and cardiovascular disease has long been reported. In specific terms, approximately $20 \%$ of patients with coronary artery disease or heart failure also have depression. The presence of depression increases the relative risk of coronary artery disease by 2.69 -fold. ${ }^{39}$ Even mild depression, such as a depressed mood, can increase the relative risk of coronary artery disease by 1.49 -fold. Among patients with coronary heart disease, unmarried people have been shown to have higher depression levels compared with the married people and patients with comorbid diseases showed a high level of depression. ${ }^{40}$ Having depression increases the relative risk of heart failure by 2.1-fold. Depression affects at least one-fifth of heart failure patients and worsens New York Heart Association classes. ${ }^{41}$

Various studies have examined the mechanisms by which depression increases the risk of coronary artery disease and heart failure. These mechanisms can be divided into the direct effect on cardiovascular systems by stress and indirect effects via lifestyle changes. Direct effects include increased serum cortisol and catecholamine levels, increases in inflammatory cytokines, platelet aggregation and oxidative stress along with endothelial dysfunction and autonomic neuropathy. It has also been suggested that the neural government of the diaphragm adversely affect emotional factors, such as psychological stress, as well as respiratory rhythms, leading to worsening of heart failure. ${ }^{41}$ Indirect mechanisms include obesity and smoking due to changes in lifestyle, failure to adhere to medication and failure to maintain adequate exercise habits. ${ }^{42}$

While restrictive measures are taken during the COVID-19 pandemic, it is important to conduct regular assessments of anxiety and depression as a component of the comprehensive management of coronary artery disease and heart failure patients, in order to detect depression tendencies. Depression needs to be identified early and appropriate treatment provided. Additionally, quitting smoking, maintaining an appropriate level of physical activity, eating a healthy diet and managing stress may be crucial in the care of patients with cardiovascular diseases.

It has been suggested that anxiety about pandemic-derived infections may delay acute coronary syndrome patients seeking help. ${ }^{43}$ It has also been reported that remote monitoring of vital signs and physical activity levels in patients with heart failure can lead to prevention of worsening heart failure and encouragement of patients for medical check-up. ${ }^{44} \mathrm{As}$ stay-at-home requests are enforced to control infection, telemedicine applications are important measures to prevent the development of cardiovascular diseases.

\section{Recommended Actions}

Following a healthy lifestyle is crucial to managing mental health in response to the COVID-19 outbreak. This includes staying in touch with family and friends, getting adequate rest, deep breathing, following a healthy diet and getting an adequate amount of physical activity and sleep, as advocated by the WHO. ${ }^{45}$ Moreover, people should obtain information from reliable sources and spend less time exposed to anxietyinducing reports in the media. Not turning to addictive substances, such as alcohol and tobacco, to relieve anxiety and stress is also important. People need to draw on skills that previously helped them overcome adversity, and they need to consult medical personnel or a counsellor when necessary. ${ }^{46} \mathrm{~A}$ healthy diet, an adequate amount of exercise and sleep and quitting smoking to improve one's immunity to maintain a healthy lifestyle is an important part of the response to COVID-19. ${ }^{47}$ In specific terms, a diet of fruits, vegetables and whole-grain foods should be consumed to optimise one's immune system and prevent obesity, and overconsumption of salt, fat and sugar should be avoided. ${ }^{48}$ Exercise is also effective in reducing the risk of cardiovascular diseases and conditions, such as locomotive syndrome, depression and dementia, and it is crucial during this pandemic.

People should engage in an exercise of adequate intensity while taking steps to protect themselves from infection by walking in places with few people or doing yoga or strength training at home. People attempting to quit smoking should receive a combination of drug and behavioural therapy at a smoking cessation clinic. Moreover, smoking cessation support needs to be available remotely through the receipt of advice using tools, such as a smoking cessation app, email, telephone or the internet. ${ }^{15}$ Typically, people temporarily gain weight after quitting smoking, and apprehensions about weight gain and worsening glucose tolerance can lead to failure to quit smoking. ${ }^{49}$ Even if such temporary weight gain is noted, it is outweighed by the reduced cardiovascular risk as a result of quitting smoking, so continuous abstinence from smoking is recommended. ${ }^{50}$

A study has reported that smokers who are highly dependent on nicotine are particularly likely to gain weight after quitting smoking. ${ }^{49}$ An intervention to deal with weight gain should be provided the same time as smoking cessation support. A combination of nicotine replacement therapy and diet and exercise will help to prevent weight gain after quitting smoking and also increase the likelihood that one successfully quits smoking. ${ }^{49}$ Furthermore, effective measures are required to mitigate negative impacts on health. This may be a way to sustain care for people living during the COVID-19 pandemic. $\square$
1. WHO. Mental health preparedness and response for the COVID-19 pandemic Report by the Director-General. Geneva: WHO, 8 January 2021. https://apps.who.int/gb/ ebwha/pdf_files/EB148/B148_20-en.pdf (accessed 11 May 2021).

2. Jenkins R. Global mental health and sustainable development 2018. BJPsych Int 2019;16:34-7. https://doi. org/10.1192/bji.2019.5; PMID: 31144683

3. UN leads call to protect most vulnerable from mental health crisis during and after COVID-19. UN, 14 May 2020. https:// news.un.org/en/story/2020/05/1063882 (accessed 9 September 2021).

4. Czeisler MÉ, Lane RI, Petrosky E, et al. Mental health, substance use, and suicidal ideation during the COVID-19 Pandemic - United States, June 24-30, 2020. MMWR Morb Mortal Wkly Rep 2020;69:1049-57. https://doi.org/10.15585/ mmwr.mm6932a1; PMID: 32790653

5. UN. Policy Brief: COVID-19 and the need for action on mental health. New York: UN, 2020. https://unsdg.un.org/sites/ default/files/2020-05/UN-Policy-Brief-COVID-19-and-mentalhealth.pdf (accessed 11 May 2021)
6. Ettman CK, Abdalla SM, Cohen GH, et al. Prevalence of depression symptoms in US adults before and during the COVID-19 pandemic. JAMA Netw Open 2020;3:e2019686. https://doi.org/10.1001/jamanetworkopen.2020.19686; PMID: 32876685.

7. Glenister KM, Ervin K, Podubinski T. Detrimental health behaviour changes among females living in rural areas during the COVID-19 pandemic. Int J Environ Res Public Health 2021;18:722. https://doi.org/10.3390/ijerph18020722; PMID: 33467693.

8. Japan Broadcasting Corporation. Suicide last month increased by $40 \%$ from last year. Women increased significantly. Corona's impact. 2020 [in Japanese].

9. Fergusson DM, Horwood LJ, Boden JM, et al. Impact of a major disaster on the mental health of a well-studied cohort. JAMA Psychiatry 2014;71:1025-31. https://doi. org/10.1001/jamapsychiatry.2014.652; PMID: 25028897.

10. Starcke K, Brand M. Decision making under stress: a selective review. Neurosci Biobehav Rev 2012;36:1228-48. https://doi.org/10.1016/j.neubiorev.2012.02.003; PMID: 22342781
11. Sinha R Chronic stress, drug use, and vulnerability to addiction. Ann N Y Acad Sci 2008;1141:105-30. https://do org/10.1196/annals.1441.030; PMID: 18991954

12. Clay JM, Parker MO. Alcohol use and misuse during the COVID-19 pandemic: a potential public health crisis? Lancet Public Health 2020:5:e259. https://doi.org/10.1016/S24682667(20)30088-8; PMID: 32277874.

13. Statistics Canada. How are Canadians coping with the COVID-19 situation? 8 April 2020. https://www150.statcan. gc.ca/n1/pub/11-627-m/11-627-m2020029-eng.htm (accessed 11 May 2021).

14. Naughton F, Ward E, Khondoker M, et al. Health behaviour change during the UK COVID-19 lockdown: findings from the first wave of the C-19 health behaviour and well-being daily tracker study. Br J Health Psychol 2021;26:624-43. https:// doi.org/10.1111/bjhp.12500; PMID: 33410229

15. Patwardhan P. COVID-19: Risk of increase in smoking rates among England's 6 million smokers and relapse among England's 11 million ex-smokers. BJGP Open 2020;4:bjgpopen20X101067. https://doi.org/10.3399/ bjgpopen20X101067; PMID: 32265183. 
16. Bommele J, Hopman P, Walters BH, et al. The double-edged relationship between COVID-19 stress and smoking: implications for smoking cessation. Tob Induc Dis 2020;18:63. https://doi.org/10.18332/tid/125580; PMID: 32733178.

17. Chen DTS. The psychosocial impact of the COVID-19 pandemic on changes in smoking behavior: evidence from a nationwide survey in the UK. Tob Prev Cessat 2020;6:59. https://doi.org/10.18332/tpc/126976; PMID: 33163705

18. Komiyama M, Ozaki Y, Miyazaki Y, et al. Short-term changes in self-rating depression scale scores after smoking cessation in neurotic patients. Intern Med 2021;60:1175-81. https://doi.org/10.2169/internalmedicine.4868-20; PMID: 33055476.

19. Tobacco Free Kids. Big tobacco is exploiting COVID-19 to market its harmful products, https:/www.tobaccofreekids. org/media/2020/2020_05_covid-marketing (accessed 4 June 2021).

20. Rowell A. Coronavirus: big tobacco sees an opportunity in the pandemic. The Conversation 14 May 2020. https:/ theconversation.com/coronavirus-big-tobacco-sees-anopportunity-in-the-pandemic-138188 (accessed 4 June 2021).

21. Giordano GN, Lindstrom M, The impact of social capital on changes in smoking behaviour: a longitudinal cohort study. Eur J Public Health 2011;21:347-54. https://doi.org/10.1093/ eurpub/ckq048; PMID: 20570962

22. López-Bueno R, Calatayud J, Casaña J, et al. CoVID-19 confinement and health risk behaviors in Spain. Front Psycho 2020;11:1426. https://doi.org/10.3389/fpsyg.2020.01426; PMID: 32581985

23. Niedzwiedz CL, Green MJ, Benzeval M, et al. Mental health and health behaviors before and during the initial phase of the COVID-19 lockdown: Iongitudinal analyses of the UK Household Longitudinal Study. J Epidemiol Community Health 2021;75:224-31. https://doi.org/10.1136/jech-2020-215060; PMID: 32978210.

24. Komiyama M, Hasegawa K. Smoking cessation as a public health measure to limit the coronavirus disease 2019 pandemic. Eur Cardiol 2020;15:e16. https://doi.org/10.15420/ ecr.2020.11; PMID: 32373189

25. WHO. Tobacco use and COVID-19. 11 May 2020. https:// www.who.int/news-room/detail/11-05-2020-who-statementtobacco-use-and-covid-19 (accessed 11 May 2021).

26. Taylor G, McNeill A, Girling A, et al. Change in mental health after smoking cessation: systematic review and metaanalysis. BMJ 2014;348:g1151. https://doi.org/10.1136/bmj. g1151; PMID: 24524926

27. Komiyama M, Yamakage H, Satoh-Asahara N, et al. Sex differences in nicotine dependency and depressive tendency among smokers. Psychiatry Res 2018:267:154-9. https://doi.org/10.1016/j.psychres.2018.06.010; PMID: 29908483

28. Stead LF, Koilpillai P, Fanshawe TR, et al. Combined pharmacotherapy and behavioural interventions for smoking cessation. Cochrane Database Syst Rev 2016:3:CD008286. https://doi.org/10.1002/14651858.CD008286.pub3; PMID: 27009521

29. Ministry of Health, Labor and Welfare. Survey report on the effects of smoking cessation treatment. 2017 [in Japanese] https://www.mhlw.go.j/file/05-Shingikai-12404000Hokenkyoku-Iryouka/0000192293.pdf (accessed 11 May 2021).

30. Cochrane Library. Coronavirus (COVID-19): effective options for quitting smoking during the pandemic. 2020. https:/ www.cochranelibrary.com/collections/doi/SC000042/ full?contentLanguage=en (accessed 11 May 2021).

31. Asiamah N, Opuni FF, Mends-Brew E, et al. Short-term changes in behaviors resulting from COVID-19-related socia isolation and their influences on mental health in Ghana. Community Ment Health J 2021;57:79-92. https://doi. org/10.1007/s10597-020-00722-4; PMID: 33033971.

32. Sidor A, Rzymski P. Dietary choices and habits during COVID-19 lockdown: experience from Poland. Nutrients 2020;12:1657. https://doi.org/10.3390/nu12061657; PMID: 32503173.

33. Himeno A, Satoh-Asahara N, Usui T, et.al. Salivary cortisol levels are associated with outcomes of weight reduction therapy in obese Japanese patients. Metabolism 2012;61:255-61. https://doi.org/10.1016/j. metabol.2011.06.023; PMID: 21871641.

34. WHO. Obesity and overweight. 2020. https://www.who.int/ news-room/fact-sheets/detail/obesity-and-overweight (accessed 11 May 2021).

35. WHO. Alcohol. 2018. https://www.who.int/news-room/factsheets/detail/alcohol (accessed 11 May 2021).

36. WHO. Implementation of WHO's global strategy to reduce the harmful use of alcohol during the first decade since its endorsement, and the way forward. Geneva: WHO; 2019

37. Hvidtfeldt UA, Tolstrup JS, Jakobsen MU, et al. Alcoho intake and risk of coronary heart disease in younger, middle-aged, and older adults. Circulation 2010;121:1589-97. https://doi.org/10.1161/CIRCULATIONAHA.109.887513; PMID: 20351238

38. Sattar N, McInnes IB, McMurray JJV. Obesity is a risk factor for severe COVID-19 infection: multiple potential mechanisms. Circulation 2020:142:4-6 https:/doi. org/10.1161/CIRCULATIONAHA.120.047659; PMID: 32320270.
39. Rugulies R. Depression as a predictor for coronary heart disease: a review and meta-analysis. Am J Prev Med 2002;23:51-61. https://doi.org/10.1016/S0749 3797(02)00439-7; PMID: 12093424

40. Eng HS, Yean LC, Das S, et al. Anxiety and depression in patients with coronary heart disease: a study in a tertiary hospital. Iran J Med Sci 2011:36:201-6. PMID: 23358115.

41. Bordoni B, Marelli F, Morabito B, et al. Depression and anxiety in patients with chronic heart failure. Future Cardiol 2018;14:115-9. https://doi.org/10.2217/fca-2017-0073; PMID: 29355040.

42. Gehi A, Haas D, Pipkin S, Whooley M. Depression and medication adherence in outpatients with coronary heart disease: findings from the Heart and Soul Study. Arch Intern Med 2005;165:2508-13. https://doi.org/10.1001/ archinte.165.21.2508; PMID: 16314548

43. Greco A, Spagnolo M, Capodanno D. Unmasking psychological reasons of delay in acute coronary syndromes presentation during the COVID-19 pandemic. Catheter Cardiovasc Interv 2021;98:407-8. https://doi.org/10.1002/ ccd 29103; PMID: 32583925

44. Bertagnin E, Greco A, Bottaro G, et al. Remote monitoring for heart failure management during COVID-19 pandemic. Int J Cardiol Heart Vasc 2021;32:100724. https://doi.org/10.1016/j. ijcha. 2021.100724: PMID: 33532544

45. UN News. COVID-19: Mental health in the age of coronavirus. 16 March 2020. https://news. un.org/en/ story/2020/03/1059542 (accessed 11 May 2021).

46. WHO. Coping with stress during the 2019-nCoV outbreak https://www.who.int/docs/default-source/coronaviruse/ coping-with-stress.pdf?sfvrsn=9845bc3a_2 (accessed 11 May 2021).

47. Kluge HHP, Wickramasinghe K, Rippin HL, et al. Prevention and control of non-communicable diseases in the COVID-19 response. Lancet 2020;395:1678-80. https://doi.org/10.1016/ S0140-6736(20)31067-9; PMID: 32401713.

48. Coelho-Ravagnani CF, Corgosinho FC, Sanches FFZ, et al. Dietary recommendations during the COVID-19 pandemic Nutr Rev 2021;79:382-93. https://doi.org/10.1093/nutrit/ nuaa067; PMID: 32653930

49. Komiyama M, Wada H, Ura S, et al. Analysis of factors that determine weight gain during smoking cessation therapy. PLoS One 2013;8:e72010. https://doi.org/10.1371/journal. pone.0072010; PMID: 23991026

50. Komiyama M, Wada $H$, Ura S, et al. The effects of weight gain after smoking cessation on atherogenic a1-antitrypsinlow-density lipoprotein. Heart Vessels 2015;30:734-9. https:// doi.org/10.1007/s00380-014-0549-9; PMID: 25086816. 